Vol. 46 (1992) [367-372]

\title{
STABILITY OF WEAK NORMAL STRUCTURE IN JAMES QUASI REFLEXIVE SPACE
}

\section{A. JiMÉNEZ-MELAdo}

\begin{abstract}
We introduce a coefficient on general Banach spaces which allows us to derive the weak normal structure for those Banach spaces whose Banach-Mazur distance to James quasi reflexive space is less than $\sqrt{3 / 2}$.
\end{abstract}

\section{INTRODUCTION}

Let $K$ be a nonempty convex subset of a Banach space $X$. The set $K$ is said to have normal structure if for each bounded convex subset $C \subset X$ consisting of more than one point there is a point $x \in C$ such that $\sup \{\|x-y\|: y \in C\}<\operatorname{diam}(C)$. We say that $X$ has weak normal structure if each nonempty convex and weakly compact subset $K \subset X$ has normal structure.

A mapping $T: K \rightarrow X$ is called nonexpansive if $\|T(x)-T(y)\| \leqslant\|x-y\|$ for all $x, y \in K$. We say that $X$ has the fixed point property (FPP) if every nonexpansive mapping $T: K \rightarrow K$ defined on a nonempty convex and weakly compact subset $K$ of $X$ has a fixed point.

A classical result of Kirk [6] states that a Banach space $X$ has the FPP whenever $X$ has weak normal structure, but Karlovitz [3] showed that this last property is not necessary. "Nonstandard" techniques were used to show that certain Banach spaces that lack weak normal structure have the FPP $[1,7,9]$. Since Lin [7] proved positive results concerning the FPP in Banach spaces with unconditional basis and the James quasi reflexive space $J$ cannot be embedded in a space with unconditional basis, it is somewhat surprising that the ideas arising from Lins's paper were used by Khamsi [4] to show that $J$ has the FPP. But Tingley [11] proved that the FPP for $J$ can be derived from Kirk's theorem [6] by showing in a direct fashion that the space $J$ has weak normal structure. Our main result shows that $J$ satisfies a stronger condition than that of Tingley.

Received 6 November 1991

Copyright Clearance Centre, Inc. Serial-fee code: 0004-9729/92 \$A2.00+0.00. 


\section{A SUFFICIENT CONDITION FOR WEAK NORMAL STRUCTURE}

We recall the following two properties each of which implies weak normal structure.

A Banach space $X$ has property $\left({ }^{*}\right)$ (see [11]) if for every weakly null (and not constant) sequence $\left(x_{n}\right)$ we have

$$
\sup _{m}\left(\underset{n}{\limsup }\left\|x_{n}-x_{m}\right\|\right)>\underset{n}{\liminf }\left\|x_{n}\right\|
$$

A Banach space $X$ with a Schauder basis has the Gossez-Lami Dozo property (in short: GLD property - see [2]) if for each $c>0$ there exists $r=r(c)>0$ such that for every $x \in X$ and every positive integer $n$ we have

$$
\left\|P_{n}(x)\right\|=1 \text { and }\left\|\left(I-P_{n}\right)(x)\right\| \geqslant c \quad \text { implies }\|x\| \geqslant 1+r
$$

where $\left(P_{n}\right)$ is the sequence of natural projections associated with the basis.

A natural generalisation of GLD can be formulated as follows. We say that a Banach space $X$ has the generalised Gossez-Lami Dozo property (GGLD property in short) if for every weakly null sequence $\left(x_{n}\right)$ such that $\lim \left\|x_{n}\right\|=1$, we have that $D\left[\left(x_{n}\right)\right]>1$, where

$$
D\left[\left(x_{n}\right)\right]=\underset{m}{\limsup }\left(\underset{n}{\limsup }\left\|x_{n}-x_{m}\right\|\right) .
$$

Since propety $\left({ }^{*}\right)$ implies weak normal structure (see [11]), the following result shows that property GGLD also implies weak normal structure.

Theorem 1. If a Banach space $X$ satisfies property GGLD then $X$ satisfies property $\left(^{*}\right)$ and then $X$ has weak normal structure.

Proof: Let $\left(x_{n}\right)$ be a weakly null sequence not constantly equal to 0 . We can suppose that $\left(x_{n}\right)$ has a subsequence $\left(x_{n}^{\prime}\right)$ such that there exists $b=\lim \left\|x_{n}\right\|$ and $b \neq 0$, because otherwise $\left({ }^{*}\right)$ is trivially satisfied.

If we put $y_{n}=x_{n}^{\prime} / b$, then we have that $\left(y_{n}\right)$ converges weakly to 0 and $\lim \left\|y_{n}\right\|=$ 1 , so that the hypothesis on property GGLD implies that $D\left[\left(y_{n}\right)\right]>1$. Hence $D\left[\left(x_{n}^{\prime}\right)\right]=$ $b D\left[\left(y_{n}\right)\right]>b=\lim \left\|x_{n}^{\prime}\right\|$ and from this the conclusion follows easily.

The converse of the above theorem is not true, since the space $X=\left(C_{0},|\cdot|\right)$, where $|x|=\|x\|_{\infty}+\sum_{i=1}^{\infty} 2^{-i}\left|x_{i}\right|$ satisfies property $\left(^{*}\right)$, but fails to have property GGLD.

In order to get stability results for GGLD in terms of the Banach-Mazur distance (Recall that for isomorphic Banach spaces $X$ and $Y$, the Banach-Mazur distance from $X$ to $Y$, denoted $d(X, Y)$, is defined to be the infimum of $\|U\|\left\|U^{-1}\right\|$ taken over 
all bicontinuous linear operators from $X$ onto $Y$ ) we define the coefficient $\beta(X)$ as the infimum of $D\left[\left(x_{n}\right)\right]$ taken over all weakly null sequences $\left(x_{n}\right)$ in $X$ such that $\lim \left\|x_{n}\right\|=1$. Obviously, $X$ has property GGLD if $\beta(X)>1$, and moreover, the following theorem shows that, in this case, property GGLD is in some sense 'contagious' under slight perturbations of the norm.

Theorem 2. If $\beta(X)>1$ and $d(Y, X)<\beta(X)$, then $Y$ has property GGLD.

Proof: By definition of the Banach-Mazur distance, there exists an isomorphism $U: X \rightarrow Y$ such that $\left\|U^{-1}\right\|=1$ and $\|U\|<\beta(X)$. Let $\left(y_{n}\right)$ be any weakly null sequence in $Y$ with $\lim \left\|y_{n}\right\|=1$. Put $x_{n}=U^{-1}\left(y_{n}\right)$ and let $\left(x_{n}^{\prime}\right)$ be a subsequence of $\left(x_{n}\right)$ such that there exists $b=\lim \left\|x_{n}^{\prime}\right\|$. For the sequence $\left(z_{n}\right)$ defined by $z_{n}=x_{n}^{\prime} / b$ we have that $\left(z_{n}\right)$ converges weakly to 0 and $\lim \left\|z_{n}\right\|=1$, so that $D\left[\left(z_{n}\right)\right] \geqslant \beta(X)$. On the other hand, $D\left[\left(z_{n}\right)\right] \leqslant(1 / b) D\left[\left(y_{n}\right)\right]$ and $\|U\| b \geqslant \lim \left\|U\left(x_{n}^{\prime}\right)\right\|=1$. Therefore, $\beta(X) \leqslant\|U\| D\left[\left(y_{n}\right)\right]$, what shows that $\beta(X) \leqslant\|U\| \beta(Y)$. It follows from this that $\beta(Y)>1$, since $\|U\|<\beta(X)$.

\section{JAMES QUASI REFLEXIVE SPACE}

Recall that the James space $J$ consists of all real sequences $x=\left(x_{n}\right)$ for which $\lim x_{n}=0$ and $\|x\|<\infty$, where

$$
\|x\|=\sup \left\{\left[\left(x_{p_{1}}-x_{p_{2}}\right)^{2}+\cdots+\left(x_{p_{m-1}}-x_{p_{m}}\right)^{2}+\left(x_{p_{m}}-x_{p_{1}}\right)^{2}\right]^{1 / 2}\right\}
$$

and the supremum is taken over all choices of $m$ and $p_{1}<p_{2}<\ldots p_{m}$. Then $J$ is a Banach space with norm $\|\cdot\|$ and the sequence $\left(e_{n}\right)$ given by $e_{n}=(0, \ldots, 0,1,0, \ldots)$, where the 1 is in the $n$th position, is a Schauder basis for $J$ (see [8]).

We need the following technical lemma.

Lемна. Let $x$ and $y$ be defined as

$$
x=\sum_{i=a}^{b} x_{i} e_{i}, \quad y=\sum_{i=c}^{d} y_{i} e_{i}
$$

with $1<a \leqslant b<c-1$ and $c \leqslant d<\infty$. Then

$$
\|x+y\|^{2} \geqslant \frac{3}{4}\left(\|x\|^{2}+\|y\|^{2}\right) .
$$

Proof: Since $\left\{i: x_{i} \neq 0\right\}$ is finite, the norm of $x$ is attained for some finite increasing sequence, say

$$
\|x\|^{2}=\sum_{i=1}^{k-1}\left(x_{p_{i}}-x_{p_{i+1}}\right)^{2}+\left(x_{p_{k}}-x_{p_{1}}\right)^{2} .
$$


If $p_{k}>b$ we would have $x_{p_{k}}=0$ and then, either $p_{1}=1$ and $\|x\|^{2}$ would be attained for the sequence $\left\{p_{1}, \ldots, p_{k-1}\right\}$, or $p_{1}>1$ and $\|x\|^{2}$ would be attained for the sequence $\left\{1, p_{1}, \ldots, p_{k-1}\right\}$. Hence we can suppose that $p_{k} \leqslant b$.

By definition of the norm on $J$, we have that

$$
\left(x_{p_{1}}-x_{p_{k}}\right)^{2} \leqslant \frac{1}{2}\|x\|^{2}
$$

and so, by (1),

$$
\sum_{i=1}^{k-1}\left(x_{p_{i}}-x_{p_{i+1}}\right)^{2} \geqslant \frac{1}{2}\|x\|^{2}
$$

The inequality $a^{2}+b^{2} \geqslant(a-b)^{2} / 2,(1)$ and (2), give

$$
\begin{aligned}
x_{p_{1}}^{2}+ & \sum_{i=1}^{k-1}\left(x_{p_{i}}-x_{p_{i+1}}\right)^{2}+x_{p_{k}}^{2} \\
& \geqslant \frac{1}{2}\left[\sum_{i=1}^{k-1}\left(x_{p_{i}}-x_{p_{i+1}}\right)^{2}+\left(x_{p_{k}}-x_{p_{1}}\right)^{2}\right]+\frac{1}{2} \sum_{i=1}^{k-1}\left(x_{p_{i}}-x_{p_{i+1}}\right)^{2} \\
& \geqslant \frac{3}{4}\|x\|^{2} .
\end{aligned}
$$

A similar argument shows that there exists a finite increasing sequence $\left\{q_{1}, \ldots, q_{r}\right\}$, with $c \leqslant q_{1} \leqslant q_{r} \leqslant d+1$, for which $\|y\|$ is attained and

$$
y_{q_{1}}^{2}+\sum_{j=1}^{r-1}\left(y_{q_{j}}-y_{q_{j+1}}\right)^{2}+y_{q_{r}}^{2} \geqslant \frac{3}{4}\|y\|^{2}
$$

Using the sequence $\left\{p_{1}, \ldots, p_{k}, b+1, q_{1}, \ldots, q_{r}, d+2\right\}$ we obtain

$$
\|x+y\|^{2} \geqslant x_{p_{1}}^{2}+\sum_{i=1}^{k-1}\left(x_{p_{i}}-x_{p_{i+1}}\right)^{2}+x_{p_{k}}^{2}+y_{q_{1}}^{2}+\sum_{j=1}^{r-1}\left(y_{q_{j}}-y_{q_{j+1}}\right)^{2}+y_{q_{r}}^{2}
$$

which with (3) and (4) gives the desired inequality.

THEOREM 3. If $X$ is any Banach space isomorphic to $J$, with $d(X, J)<\sqrt{3 / 2}$ then $X$ satisfies property GGLD.

Proof: By Theorem 2, we only need to prove that $\beta(J) \geqslant \sqrt{3 / 2}$. Let $\left(x_{n}\right)$ be any weakly null sequence in $J$ with $\lim \left\|x_{n}\right\|=1$. Then there exists a subsequence $\left(x_{n}^{\prime}\right)$ of $\left(x_{n}\right)$ and a sequence $\left(u_{n}\right)$ in $J$ such that
(i) $\lim \left\|x_{n}^{\prime}-u_{n}\right\|=0$
(ii) $u_{n}=\sum_{i=e_{n}}^{b_{n}} u_{n}(i) e_{i}$ 
with $a_{n} \leqslant b_{n}<a_{n+1}-1$ for every positive integer $n$.

Applying the lemma to the sequence $\left(u_{n}\right)$, we get

$$
\left\|u_{n}+u_{m}\right\|^{2} \geqslant \frac{3}{4}\left(\left\|u_{n}\right\|^{2}+\left\|u_{m}\right\|^{2}\right) \quad(n \neq m),
$$

and then, by $(i)$ and $(6), D\left[\left(x_{n}^{\prime}\right)\right]=D\left[\left(u_{n}\right)\right] \geqslant \sqrt{3 / 2}$. Since $D\left[\left(x_{n}\right)\right] \geqslant D\left[\left(x_{n}^{\prime}\right)\right]$ and $\left(x_{n}\right)$ is arbitrary, we have $\beta(J) \geqslant \sqrt{3 / 2}$.

REMARK 1. By Theorem 3, a Banach space $X$ has weak normal structure whenever $d(X, J)<\sqrt{3 / 2}$. This result is sharp because if we renorm the space $J$ according to

$$
|x|=\max \left\{\|x\|, \sqrt{3} \sup _{i<j}\left|x_{i}-x_{j}\right|\right\}
$$

we have that $\|x\| \leqslant|x| \leqslant \sqrt{3 / 2}\|x\|$ and $(J,|\cdot|)$ lacks weak normal structure. Indeed, the sequence $\left(x_{n}\right)$ given by $x_{n}=e_{3 n+1}-e_{3 n+2}$ is a diametral sequence in $(J,|\cdot|)$, from which the result follows (see, for example, [10]).

REMarK 2. Khamsi [5] associated with any Banach space $X$ with a finite dimensional Schauder decomposition the coefficient $\beta_{p}(X)$ defined for $p \in[1, \infty)$ as the infimum of the set of numbers $\lambda$ such that

$$
\left(\|x\|^{p}+\|y\|^{p}\right)^{1 / p} \leqslant \lambda\|x+y\|
$$

for every $x$ and $y$ in $X$ with $\operatorname{supp}(x)<\operatorname{supp}(y)$.

He showed that a Banach space $X$ has weak normal structure whenever $\beta_{p}(X)<2^{1 / p}$ for some $p \in[1, \infty)$. But for the decomposition given by the usual basis in $J$ we have that $\beta_{p}(J) \geqslant 2^{1 / p}$ for all $p \in[1, \infty)$.

Moreover, it is not hard to see that $\beta_{p}(X) \beta(X) \geqslant 2^{1 / p}$ for all $p \in[1, \infty)$ and for all finite dimensional decompositions of $X$ and hence $\beta(X)>1$ if $X$ has a finite dimensional decomposition such that $\beta_{p}(X)<2^{1 / p}$ for some $p \in[1, \infty)$.

Since $\beta(J)=\sqrt{3 / 2}$, this leads to the following question suggested by Khamsi (personal communication): Does the space $J$ have a basis (or a finite dimensional decomposition) such that $\beta_{p}(J)<2^{1 / p}$ for some $p \in[1, \infty)$ ?

\section{REFERENCES}

[1] J.M. Borwein and B. Sims, 'Nonexpansive mappings on Banach lattices and related topics', Houston J. Math. 10 (1984), 339-355.

[2] J.P. Gossez and E. Lami Dozo, 'Structure normale et base de Schauder', Bull. Acad. Roy. Belgique 15 (1969), 673-681. 
[3] L.A. Karlovitz, 'Existence of fixed points for nonexpansive mappings in a space without normal structure', Pacific J. Math. 66 (1976), 153-159.

[4] M.A. Khamsi, 'James quasi reflexive space has the fixed point property', Bull. Austral. Math. Soc. 38 (1989), 25-30.

[5] M.A. Khamsi, 'Normal structure for Banach spaces with Schauder decomposition', Canad. Math. Bull. 32 (1989), 344-351.

[6] W.A. Kirk, 'A fixed point theorem for mappings which do not increase distances', Amer. Math. Monthly 72 (1965), 1004-1006.

[7] P.K. Lin, 'Unconditional bases and fixed points of nonexpansive mappings', Pacific J. Math. 116 (1985), 69-76.

[8] J. Lindenstrauss and L. Tzafriri, Classical Banach spaces, Vol I (Springer-Verlag, Berlin, Heidelberg, New York, 1977).

[9] B. Maurey, 'Points fixes des contractions sur un convexe ferme de $L_{1}$ ', in Seminaire d'analyse fonctionelle (Ecole Polytechnique, Palaiseau, Exposê No. VIII, 1980/1981).

[10] S. Swaminathan, 'Normal structure in Banach spaces and its generalizations', Contemp. Math. 18, 201-215. A.M.S., Providence, R.I. .

[11] D. Tingley, 'The normal structure of James quasi reflexive space', Bull. Austral. Math. Soc. 42 (1990), 95-100.

Department of Análisis Matemático

Universided de Málage

Facultad de Ciencias

29071 Málaga

Spain 